Molecular Physics, 1983, Vol. 49, No. 4, 899-912

\title{
Excited state energetics of aniline-rare-gas van der Waals complexes
}

\author{
by AVIV AMIRAV, UZI EVEN and JOSHUA JORTNER \\ Department of Chemistry, Tel Aviv University, \\ 69978 Tel Aviv, Israel \\ and B. DICK \\ Department of Chemistry, University of Philadelphia, \\ Philadelphia, Pennsylvania 19104, U.S.A.
}

(Received 10 November 1982 ; accepted 23 February 1983)

\begin{abstract}
Aniline $-\mathrm{Ne}_{n}$ and aniline- $\mathrm{Ar}_{n}(n=1$ and 2) complexes were synthesized in continuous and in pulsed supersonic expansions and interrogated by laserinduced fluorescence. Information on the formation kinetics and excited state energetics was obtained.
\end{abstract}

\section{INTRODUCTION}

There has been considerable experimental and theoretical activity aimed towards the understanding of excited state energetics and dynamics of large van der Waals molecules [1-12], consisting of aromatic molecules, for example, benzene $[11,12]$, alkylbenzenes $[10]$, fluorene $[5,6]$, anthracene [7], tetracene $[1,2]$ and pentacene $[3]$, bound to rare-gas atoms. Such van der Waals complexes can be viewed as a large molecule embedded in a well characterized solvent structure, whose composition can adequately be specified. Accordingly, solvent effects of excited state energetics and dynamics can be explored from the microscopic point of view [2,9]. In this paper we continue the programme [1-6] of the characterization of van der Waals molecules and the exploration of microscopic solvation effects on excited state energetics of large van der Waals complexes, reporting the results of an experimental study of the formation kinetics of aniline- $\operatorname{Ar}_{n}(n=1$ and 2$)$ as well as the microscopic spectral shifts of aniline- $\mathrm{Ne}_{n}(n=1$ and 2$)$ and aniline- $\mathrm{Ar}_{n}(n=1$ and 2$)$ complexes synthesized in supersonic expansions and interrogated by laser-induced fluorescence. The $S_{0} \rightarrow S_{1}$ transitions of the van der Waals complexes of aniline with rare gases provide two novel features of microscopic solvent shifts. First, the spectral shifts for aniline- $\mathrm{Ar}_{n}$ reported herein, together with the spectroscopic data for toluene-Ar ${ }_{n}$ obtained by Smalley and Hopkins [10] and for benzene-Ar published by Schlag et al. $[11,12]$, will demonstrate the enhancement of the excited state dispersive stabilization energy by increasing the oscillator strength for the $S_{0} \rightarrow S_{1}$ transition in the series of substituted benzenes. Secondly, the aniline molecule undergoes an appreciable change in its dipole moment upon $S_{0} \rightarrow S_{1}$ excitation, the dipole moment being 1.53 Debye (Debye $\approx 3.33564 \mathrm{C} \mathrm{m}$ ) in its $S_{0}$ ground state and 2.38 Debye in the $S_{1}$ excited electronic configuration 
[13]. It would be interesting to explore the excited state energetics of a van der Waals complex, whose molecular component undergoes a considerable change in its dipole moment upon electronic excitation. This large change in the electronic charge distribution will quantitatively modify the magnitude of the microscopic spectral shift for aniline- $R_{n}(R=\mathrm{Ne}$ and Ar $)$ complexes, resulting in a new contribution originating from dipole-induced-dipole interactions (DIDI). This DIDI contribution is absent in spectral shifts of complexes consisting of non-polar alternant hydrocarbons bound to rare-gas atoms and provides a new contribution to the stabilization of the $S_{1}$ state in van der Waals complexes containing a polar aromatic component. On the basis of model calculations of the DIDI, we shall assert that the contribution of DIDI to the large spectral shifts of aniline- $R_{n}$ complexes is modest, not exceeding 10 per cent of the dispersive stabilization, whereupon the spectral shifts are dominated by dispersive interactions.

\section{EXPERIMENTAL DETAILS}

Laser-induced fluorescence spectroscopy in supersonic expansions of $\mathrm{Ne}$, $\mathrm{Ar}, \mathrm{Kr}$ and $\mathrm{Xe}$ seeded with aniline was conducted using both continuous and pulsed jets. Two types of continuous nozzle were used, a $50 \mu \mathrm{m}$ hole in a $50 \mu \mathrm{m}$ disc and a $150 \mu \mathrm{m}$ hole in a $100 \mu \mathrm{m}$ disc, which were attached to a stainless steel sample chamber. The pulsed nozzle with a diameter of $D=600 \mu \mathrm{m}$ employed a solenoid-activated mechanical valve. The temporal shape of the gas pulse (full width at half maximum) was $200 \mu \mathrm{s}$ and the repetition rate was $10 \mathrm{~Hz}$ [14]. The diluent gas was bubbled through liquid aniline contained in a metal container in the vacuum system which was located just before the sample chamber. The container and the sample chamber were maintained at the same temperature. Experiments were performed with the container and the sample chamber kept at $25^{\circ} \mathrm{C}$ (aniline vapour pressure 0.3 Torr) and at $70^{\circ} \mathrm{C}$ (aniline vapour pressure to 10 Torr). The pumping system of the supersonic beam apparatus consisted of a $4^{\prime \prime}$ diffusion pump (CVC) backed by two mechanical pumps (Sergant-Welch pump with a pumping speed of $500 \mathrm{dm}^{3} \mathrm{~min}^{-1}$ and an Edwards pump with a pumping speed of $200 \mathrm{dm}^{3} \mathrm{~min}^{-1}$ ). Light from a pulsed nitrogen pumped dye laser (Molectron DL2) with a spectral width of $0.3 \mathrm{~cm}^{-1}$ was frequency doubled by a phase matched KDP crystal, the averaged pulse output power of the light in the spectral region $2880-3000 \AA$ being $2-4 \mu \mathrm{W}$. The laser beam crossed the continuous supersonic expansion at distances of $X=5-8 \mathrm{~mm}$ and the pulsed expansion at $X=15 \mathrm{~mm}$ down the nozzle. The laser-induced fluorescence spectrum corresponds to the intensity of the total fluorescence versus the laser wavelength, which was continuously scanned. The total fluorescence in the range $2950-4000 \AA$ was collected by focusing the emitted radiation by a quartz lens on a photomultiplier and recorded by a boxcar integrator after normalization to the laser intensity.

\section{EXPERIMENTAL RESULTS}

\subsection{Cooling of aniline in supersonic expansions}

Figure 1 shows the fluorescence excitation spectrum in the spectral range $2880-2980 \AA$ of aniline in continuous supersonic expansions of Ar at moderately low stagnation pressures of $p=200$ Torr and $p=500$ Torr. The pronounced 


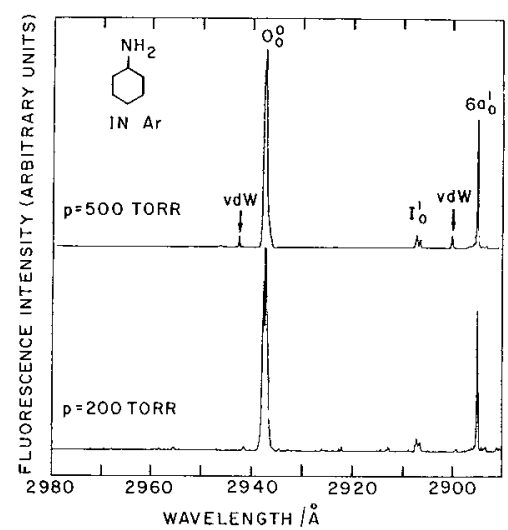

Figure 1. Fluorescence excitation spectra in the range $2890-2980 \AA$ of aniline in continuous supersonic expansions of $\mathrm{Ar}$. Aniline at $70^{\circ} \mathrm{C}$ (vapour pressure 10 Torr) was mixed with $\mathrm{Ar}$ at $p=200$ Torr (lower curve) and at $p=500$ Torr (upper curve). The seeded gas was expanded through a $150 \mu \mathrm{m}$ nozzle at $70^{\circ} \mathrm{C}$. The laser crossed the supersonic expansion at $7 \mathrm{~mm}$ down the nozzle. The prominent vibrational features $0_{0}{ }^{0}, 6 a_{0}{ }^{1}$ and $I_{0}{ }^{1}$ are marked. Spectral features due to aniline-Ar van der Waals complexes are marked vdW.

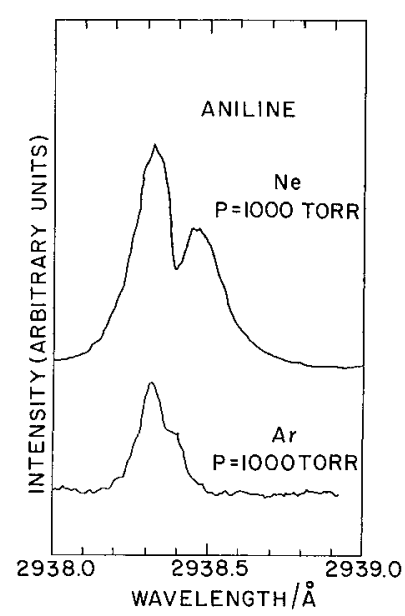

Figure 2. Fluorescence excitation spectra of the electronic origin of aniline in pulsed supersonic expansions of $\mathrm{Ne}$ and Ar. Aniline at $25^{\circ} \mathrm{C}$ (vapour pressure $0.3 \mathrm{Torr}$ ) was seeded into the rare gas at $p=1000$ Torr and expanded through the $600 \mu \mathrm{m}$ nozzle. The laser crossed the jet at $x=15 \mathrm{~mm}$ downstream.

spectral features, whose intensities are practically invariant to the change in the stagnation pressure are the electronic origin $\left(0_{0}{ }^{0}\right)$, the $I_{0}{ }^{1}$ transition and the $6 a_{0}{ }^{1}$ transition $[15,16]$, which are marked in figure 1 . The overall vibrational structure of the cold aniline molecule is in agreement with the previous results of Mikami et al. [17] and of Smalley et al. [18, 19]. The electronic origin reveals a double-humped structure consisting of two peaks, which is characteristic of a rotational envelope for a $B$-type rotational contour. Figure 2 shows medium resolution laser-induced fluorescence spectra of the electronic origin of aniline 
Table 1. Internal temperatures of aniline in supersonic expansions of $\mathrm{Ne}$ and $\mathrm{Ar}$.

\begin{tabular}{cccccc}
\hline Type of jet & Diluent & $P /$ Torr & $T_{\mathrm{t}} / \mathrm{K}$ & $T_{\mathrm{R}} / \mathrm{K}$ & $T_{\mathrm{V}} / \mathrm{K}$ \\
\hline $\begin{array}{c}\text { Pulsed } \\
D=600 \mu \mathrm{m} \\
\quad \begin{array}{l}\text { Pulsed } \\
D=600 \mu \mathrm{m}\end{array}\end{array}$ & $\mathrm{Ne}$ & 1000 & 0.31 & $9 \pm 1$ & - \\
$\begin{array}{l}\text { Continuous } \\
D=150 \mu \mathrm{m}\end{array}$ & Ar & 1000 & 0.39 & $2 \cdot 2 \pm 0.2$ & - \\
$\begin{array}{c}\text { Continuous } \\
D=150 \mu \mathrm{m}\end{array}$ & Ar & 200 & 3.9 & $(20)$ & 20 \\
\hline
\end{tabular}

in pulsed jets of $\mathrm{He}, \mathrm{Ne}$ and $\mathrm{Ar}$. Our spectral resolution of $0.3 \mathrm{~cm}^{-1}$ makes it possible to resolve the rotational contours of aniline, while previous work of Mikami et al. [17], who used a lower spectral resolution of $3 \mathrm{~cm}^{-1}$, could not accomplish this goal.

A quantitative characterization of the internal cooling of aniline in jets was provided by the evaluation of the translational, rotational and vibrational temperatures. The terminal translational temperature $T_{\mathrm{t}}$ was calculated (table 1) from the relation [20] $T_{\mathrm{t}}=T_{0}\left[1+\frac{1}{2}(\gamma-1) M^{2}\right]^{-1}$ where the Mach number is [20] $M=A(\mathrm{pD} / \mathrm{atm} \mathrm{cm})^{0 \cdot 4}$ with $A=148$ for Ne [21] and $A=133$ for $\operatorname{Ar}[20,21]$. The rotational temperatures $T_{\mathrm{R}}$ in pulsed jets were determined by fitting the rotational contours of figure 2 by computer-simulated contours of aniline [22], which were generated using the asymmetric-rotor computer programme of Birss and Ramsay $[23,24]$ and utilizing the rotational constants of Christoffersen $e t$ al.

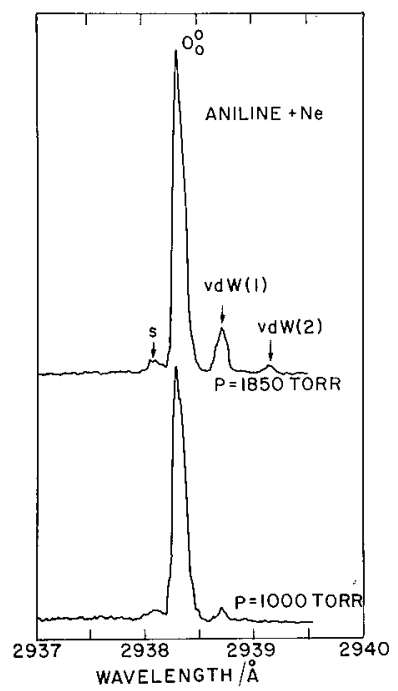

Figure 3. Fluorescence excitation spectra of aniline in pulsed jets of $\mathrm{Ne}$. Aniline at $25^{\circ} \mathrm{C}$ (vapour pressure 0.3 Torr) was seeded into $\mathrm{Ne}$ at the stagnation pressures indicated on the curves and expanded through a $600 \mu \mathrm{m}$ nozzle. The laser crossed the jet at $x=15 \mathrm{~mm}$. The electronic origin of bare aniline is marked by $0_{0}{ }^{\circ}$, while the spectral features marked $\mathrm{vdW}(1)$ and $\mathrm{vdW}(2)$ correspond to van der Waals complexes. 
[25]. Rough estimates of $T_{\mathrm{R}}$ were obtained for continuous jets of Ar from the widths (full width at half maximum) of the high resolution spectra, which were confronted with the widths of computer-simulated contours [22]. From the rotational temperatures presented in table 1 , we note that the efficiency of rotational cooling varies in the order $\mathrm{Ne}<\mathrm{Ar}$, reflecting the manifestation of the velocity slip effect [26], which prevails for the lighter diluent. Estimates of the vibrational temperatures $T_{\nabla}$ was obtained from hot band spectroscopy. In addition to the three prominent vibrational features originating from excitations from the vibrational origin of the ground $S_{0}\left({ }^{1} A_{1}\right)$ state, the laser-induced fluorescence spectrum at $p=200$ Torr of $\operatorname{Ar}$ (figure 1 ) reveals several additional weak features, whose intensity relative to the $0_{0}{ }^{0}$ band is $\leqslant 0 \cdot 05$, which originate from hot bands. From the intensity ratio $J$ between the $I_{1}{ }^{1}$ and $0_{0}{ }^{0}$ transitions, we estimate the vibrational temperature from the relation $T_{V}=-\left(\hbar \omega_{1} / k_{\mathrm{B}}\right) \ln J$, where $\hbar \omega_{1}=40 \cdot 8 \mathrm{~cm}^{-1}$ is the ground state vibrational frequency of the inverse vibration [27]. At $p=200$ Torr $A r$ we find $J=0.05$ and estimate the vibrational temperature to be $T_{V}=20 \mathrm{~K}$. At $p=500$ Torr all the hot bands of the bare molecule are suppressed (figure 1 ) and $J<0 \cdot 01$, so that we estimate $T_{V}<12 \mathrm{~K}$. From the data summarized in table 1 , we note that at a constant value of $\mathrm{pD}$ the order of internal temperatures is $T_{\mathrm{t}}<T_{\mathrm{R}} \lesssim T_{\mathrm{V}}$, as is expected for axisymmetric jets [22].

\subsection{Aniline-Ne van der Waals complexes}

The laser-induced fluorescence spectrum of aniline in a pulsed jet of $\mathrm{Ne}$ at $p=1000$ Torr exhibits two weak satellites in the vicinity of the $0_{0}{ }^{0}$ transition of the bare molecule (figure 3 ). These involve a blue-shifted band labelled $(s)$ at $+2.6 \mathrm{~cm}^{-1}$ and a red shifted band at $-4.8 \mathrm{~cm}^{-1}$, relative to the $0_{0}{ }^{0}$ origin. These spectral shifts, as well as all other spectral shifts reported in this paper, refer to the energy of the maximum of the spectral feature relative to the maximum of the $0_{0}{ }^{0}$ transition, which corresponds to the peak of the $R$ branch of the rotational envelope. As is apparent from figure 3 , the intensity of the $(s)$ blue satellite is independent of the stagnation pressure. Accordingly, this weak feature cannot be attributed to either a hot band of the bare molecule or to a van der Waals complex. The $(s)$ feature may be due to the $0_{0}{ }^{0}$ transitions of isotopically substituted ${ }^{13} \mathrm{C}^{12} \mathrm{C}_{5} \mathrm{H}_{5} \mathrm{NH}_{2}$ molecules, whose overall abundance is 6 per cent. The spectral shift of the $s$ band is of the correct magnitude, being close to the ${ }^{13} \mathrm{C}$ isotopic shift determined by two-photon ionization of aniline in jets [28]. The assignment of the $(s)$ band is plausible but by no means conclusive, as a contribution from an unidentified impurity cannot be ruled out. The spectral feature marked vdW(1) on the low-energy side of the $0_{0}{ }^{0}$ transition of the bare molecule is attributed to the $0-0 \quad S_{0} \rightarrow S_{1}$ excitation of the aniline- $\mathrm{Ne}_{1}$ complex. The additional red-shifted spectral feature marked vdW(2), which is exhibited at a higher stagnation pressure (figure 3 ), is assigned to the electronic origin of the aniline- $\mathrm{Ne}_{2}$ complex. Two qualitative diagnostic methods were utilized for the identification and characterization of the spectral features, which correspond to the vibrationless electronic excitation of aniline- $\mathrm{Ne}_{n}(n=1$ and 2) complexes.

(a) The dependence of the energies of the spectral features on the nature of the diluent. These two spectral features appear only in $\mathrm{Ne}$. 
(b) The order of appearance of the spectral features with increasing stagnation pressure. The higher energy peak is exhibited first. The intensity of the $\mathrm{vdW}(1)$ feature exhibits the dependence of the form $p^{2}$ on the stagnation pressure, as appropriate for aniline- $\mathrm{Ne}_{1}$ produced via three-body collisions. At higher pressures the aniline- $\mathrm{Ne}_{2}$ excitation is exhibited. We are thus led to the assignment of the spectral features summarized in table 2 .

Table 2. Energetics of the electronic origin of aniline- $\mathrm{Ne}_{n}$ molecules.

\begin{tabular}{cccc}
\hline Band label & Wavelength $/ \AA+$ & $\begin{array}{c}\delta \nu \\
\text { Spectral shift } / \mathrm{cm}^{-1} \ddagger\end{array}$ & Assignment \\
\hline 0 & 2938.31 & 0 & Aniline $0_{0}{ }^{0}$ \\
$\operatorname{vdW}(1)$ & 2938.72 & -4.8 & ${\text { Aniline }-\mathrm{Ne}_{1}}$ \\
$\operatorname{vdW}(2)$ & 2939.14 & -9.6 & Aniline- $\mathrm{Ne}_{2}$ \\
\hline
\end{tabular}

† Wavelengths refer to peaks of spectral features. Absolute accuracy of wavelength scale is $\pm 0.5 \AA\left( \pm 6 \mathrm{~cm}^{-1}\right)$. Relative accuracy of wavelength scale is $\pm 0.04 \AA$ $\left( \pm 0.5 \mathrm{~cm}^{-1}\right)$.

$\ddagger$ The spectral shifts are accurate within $\pm 1 \mathrm{~cm}^{-1}$.

\subsection{Aniline-Ar van der Waals complexes}

Figure 1 shows that when efficient internal vibrational and rotational cooling has been attained new spectral features appear on the low energy side of the $0_{0}{ }^{0}$ transition and of the $6 a_{0}{ }^{1}$ transition (marked by arrows in figure 1), which are attributed to van der Waals complexes of aniline with Ar. The spectral feature marked vdW on the low energy side of the $0_{0}{ }^{0}$ transition of the bare molecule is assigned to the vibrationless $0-0 S_{0} \rightarrow S_{1}$ excitation of an aniline-Ar complex, while the spectral feature marked $\mathrm{vdW}$ on the low energy side of the $6 a_{0}{ }^{1}$ transition is attributed to the electronic vibrational excitation of an aniline-Ar complex with the aniline molecule being excited by one quantum of the $6 a$ vibration in the $S_{1}$ state. As is apparent from figure 4 , two spectral features peaking at $2942.5 \AA$ and at $2946.4 \AA$ appear with increasing stagnation pressure $p$. On the basis of the diagnostic criteria $(a)$ and $(b)$ of $\$ 3 \cdot 2$, we are led to the assignment of the spectral features summarized in table 3 as originating from the vibrationless $0-0 S_{0} \rightarrow S_{1}$ excitation of the aniline- $\mathrm{Ar}_{1}$ and aniline- $-\mathrm{Ar}_{2}$ van der Waals molecules.

Quantitative support for the spectroscopic identification of the spectral features of the aniline- $\operatorname{Ar}_{n}(n=1$ and 2$)$ van der Waals molecules was obtained from the following diagnostic methods.

(c) The reduction of the relative intensity of the bare molecule with increasing stagnation pressure. Quantitative data regarding the complexation of the aniline molecule by rare gases in continuous jets are presented in $\$ 3.4$.

(d) The formation kinetics of the aniline- $\mathrm{Ar}_{1}$ complex. In $\S 3.4$ we shall provide quantitative kinetic data for the rise and fall of the spectral feature of aniline-A $r_{1}$ with increasing $p$ in continuous jets. These kinetic data provide conclusive evidence for the assignment of this spectral feature to the aniline-Ar complex. 


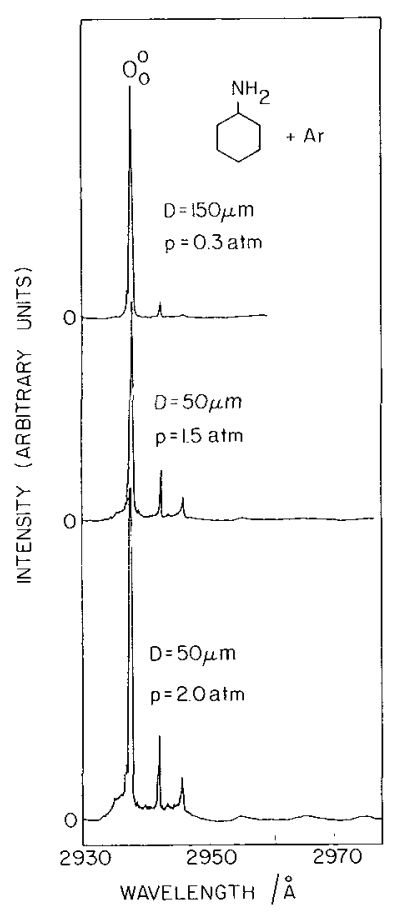

Figure 4. Fluorescence excitation spectra in the range 2930-2970 $\AA$ of aniline in continuous supersonic expansions of Ar. Aniline at $70^{\circ} \mathrm{C}$ (vapour pressure 10 Torr) was mixed with $\mathrm{Ar}$ at the pressures indicated on the figure. The seeded gas was expanded through a nozzle at $70^{\circ} \mathrm{C}$. Upper curve: $p=0.3 \mathrm{~atm} ; D=150 \mu \mathrm{m}$. Middle curve : $p=1.5 \mathrm{~atm} ; D=50 \mu \mathrm{m}$. Lower curve : $p=2.0 \mathrm{~atm} ; D=50 \mu \mathrm{m}$. The electronic origin of the bare molecule is marked $0_{0}{ }^{0}$.

Table 3. Energetics of the electronic origin of aniline- $-\mathrm{Ar}_{n}$ van der Waals molecules.

\begin{tabular}{cccc}
\hline Band label & Wavelength $/ \AA \dagger$ & $\begin{array}{c}\delta \nu \\
\text { Spectral shift } / \mathrm{cm}^{-1} \ddagger\end{array}$ & Assignment \\
\hline 0 & 2938.30 & 0 & Aniline $0_{0}{ }^{0}$ \\
$\operatorname{vdW}(1)$ & 2943.30 & -58 & Aniline-Ar \\
$\operatorname{vdW}(2)$ & 2947.15 & -102 & Aniline-Ar \\
\hline
\end{tabular}

$\uparrow$ Absolute accuracy of wavelength scale is $\pm 0.5 \AA\left( \pm 6 \mathrm{~cm}^{-1}\right)$. Relative accuracy of wavelength scale is $\pm 0.1 \AA\left( \pm 1.0 \mathrm{~cm}^{-1}\right)$.

$\ddagger$ The spectral shifts are accurate within $\pm 2 \mathrm{~cm}^{-1}$.

\subsection{Complexing of aniline by $\mathrm{Ar}, \mathrm{Kr}$ and $\mathrm{Xe}$}

We have followed the reduction of the intensity $[A]$ of the $0_{0}{ }^{0}$ spectral feature of the bare aniline molecule in supersonic expansions of $\mathrm{Ar}, \mathrm{Kr}$ and $\mathrm{Xe}$, with increasing the stagnation pressure $p$ of the diluent, while the aniline pressure was constant. Figure 5 demonstrates that the intensity of the bare molecule obeys the relation $[A] \propto \exp \left(-K_{1} p^{2}\right)$. Thus, the attachment of the first rare-gas 


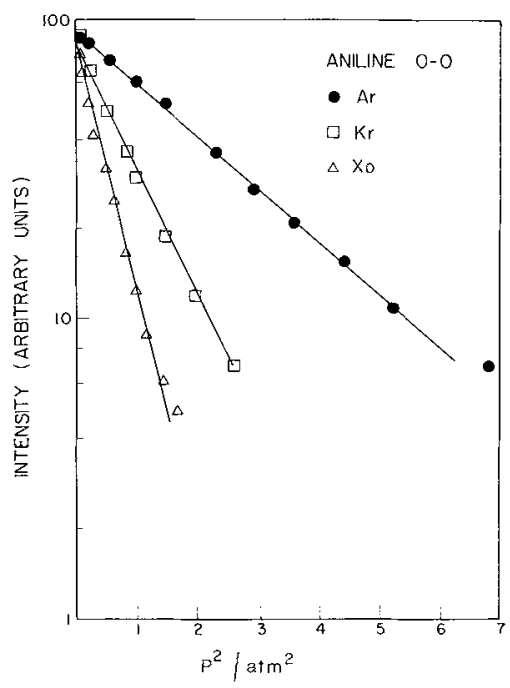

Figure 5. The dependence of the peak intensity of the $0_{0}{ }^{\circ}$ transition of the bare aniline molecule on the stagnation pressure $p$ of rare-gas diluents in continuous jets. Aniline at a constant vapour pressure of 3 Torr was mixed with $\mathrm{Ar}, \mathrm{Kr}$ or $\mathrm{Xe}$ and expanded through a $50 \mu \mathrm{m}$ nozzle maintained at $70^{\circ} \mathrm{C}$. The relative intensities are presented in arbitrary units for each diluent.

atom $R$ to the aniline molecule proceeds via a three-body collision

$$
\text { Aniline }+R+R \stackrel{K_{1}}{\longrightarrow} \text { Aniline }-R_{1}+R,
$$

where $K_{1}$ is the effective three-body recombination rate. Such a three-body recombination mechanism was observed previously for the binding of rare-gas atoms to diatomics, such as iodine [29] and to very large aromatic molecules, for instance, tetracene [2] and pentacene [3]. The relative three-body recombination rate constants for aniline with rare gases (for a $D=50 \mu$ nozzle at $343 \mathrm{~K}$ ) are $0.39 \mathrm{~atm}^{-2}$ for $\mathrm{Ar}, 0.99 \mathrm{~atm}^{-2}$ for $\mathrm{Kr}$ and $1.84 \mathrm{~atm}^{-2}$ for Xe. The relative values of the recombination cross sections $\sigma_{0}$ which are given by the relation [30] $K_{1} \propto \sigma_{0}{ }^{5 / 2}$ are 1.0 for $\mathrm{Ar}, 1.45$ for $\mathrm{Kr}$ and 1.85 for Xe. These cross sections show the same trend as exhibited by the relative $\sigma_{0}$ values for the three-body relative cross sections for the recombination of rare gases with tetracene [2], establishing some universal features of the three-body recombination of medium- and large-sized molecules with rare gases.

\subsection{Formation and disappearance of kinetics of aniline-Ar $r_{1}$}

The kinetic data of $\$ 3.3$ provide evidence that the aniline- $R_{1}$ van der Waals complexes are produced by a three-body collision. In the case of the Ar diluent, the disappearance of the bare molecule with increasing $p$ is accompanied by the appearance of two spectral features, $\operatorname{vdW}(1)$ and $\mathrm{vdW}(2)$, according to the labelling of table 3 . We have monitored the dependence of the intensity of band $\operatorname{vdW}(1)$ on the stagnation pressure. The intensity data of figure 6 can be accounted for by assigning band $\mathrm{vdW}(1)$ to the aniline-Ar ${ }_{1}$ complex, which is produced via reaction (3.1), and is destroyed by the three-body collision

$$
\text { Aniline- } \mathrm{Ar}_{1}+\mathrm{Ar}+\mathrm{Ar} \stackrel{K_{2}}{\longrightarrow} \text { Aniline--Ar }+\mathrm{Ar} \text {. }
$$




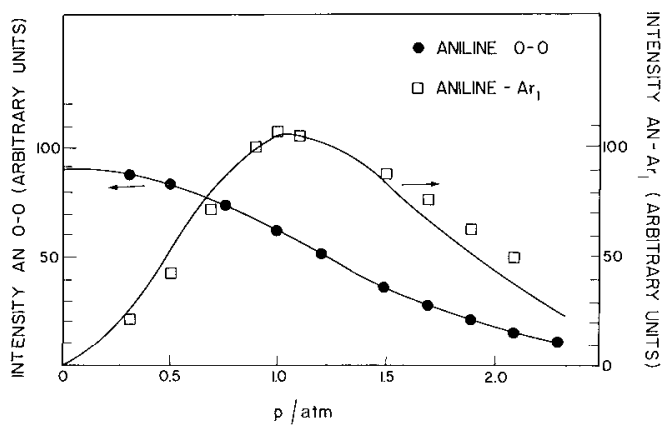

Figure 6. The dependence of the intensity of the electronic origin of the bare aniline molecule (AN $0-0)$ and of the intensity of the vibrationless transition of aniline-Ar $\left(\mathrm{AN}-\mathrm{Ar}_{1}\right.$ ) on the stagnation pressure $p$ of the Ar diluent in continuous jets. The intensities of the bare molecule and of the aniline- $\mathrm{Ar}_{1}$ complex are presented in arbitrary units for each spectral feature. The solid curves are calculated from (3.3) and (3.4) with $K_{1}=0.39 \mathrm{~atm}^{-2}$ and $K_{2} / K_{1}=4.5$.

The concentrations of the bare molecule and the complex are

$$
\begin{gathered}
{[\text { Aniline }]=[\mathrm{A}]_{0} \exp \left(-K_{1} p^{2}\right)} \\
{\left[\text { Aniline }-\mathrm{Ar}_{1}\right]=[A]_{0}\left[K_{1} /\left(K_{1}-K_{2}\right)\right]\left[\exp \left(-K_{2} p^{2}\right)-\exp \left(-K_{1} p^{2}\right)\right] .}
\end{gathered}
$$

A reasonable fit of the experimental data was obtained with $K_{1}=0.39 \mathrm{~atm}^{-2}$ and $K_{2} / K_{1}=4.5$ (figure 6). The enhancement of the rate for the formation of aniline- $-\mathrm{Ar}_{2}$ relative to the formation rate of aniline-Ar $\mathrm{Ar}_{1}$ is similar to that observed for the rates of formation of tetracene- $\mathrm{Ar}_{2}$ and of tetracene- $\mathrm{Ar}_{1}[2]$. The present analysis provides conclusive kinetic evidence for the identification of band $\mathrm{vdW}(1)$ (of table 3 ) to correspond to the aniline-Ar $\mathbf{r}_{1}$ complex. On the basis of the order of appearance of band $\mathrm{vdW}(2)$, it is assigned to the aniline- $\mathrm{Ar}_{2}$ complex.

\subsection{Some negative results}

We were able to identify two well defined, distinct, spectral features, which correspond to the aniline- $\operatorname{Ar}_{n}(n=1$ and 2) van der Waals molecules. When the stagnation pressure of $\mathrm{Ar}$ was further increased from $1.0 \mathrm{~atm}$ up to $p_{0}=2.5 \mathrm{~atm}$, no new distinct spectral features corresponding to higher aniline- $\mathrm{Ar}_{n}$ complexes with $n \geqslant 3$ could be observed in the laser-induced fluorescence spectrum. Our failure to observe the spectral features of higher $n \geqslant 3$ complexes of Ar can be traced to one or several of the following causes.

(1) Low efficiency of formation of such $(n \geqslant 3)$ complexes.

(2) Low fluorescence quantum yields for emission from these $(n \geqslant 3)$ complexes.

(3) The existence of a large number of chemical isomers for each aniline- $\mathrm{Ar}_{n}$ complex with a fixed coordination number $n \geqslant 3$. Each chemical isomer is expected to exhibit a different spectral shift, so that the spectra of these higher complexes are expected to be broad and structureless.

The kinetic effect $(1)$ is inconsistent with the observation reported in $\S 3.4$, that is, in the pressure range $p=1 \cdot 0-2.5$ atm of Ar effective complexation of the 
aniline molecule is exhibited. The possibility of the enhancement of the intramolecular electronic relaxation in aniline- $\mathrm{Ar}_{n}$ complexes, effect (2), implies that the decay lifetimes of the $S_{1}$ state of aniline- $\mathrm{Ar}_{1}$ and of aniline-Ar $\mathrm{r}_{2}$ are considerably shorter than the lifetime of the $S_{1}$ state of bare aniline. We have monitored the radiative decay lifetimes of aniline- $\mathrm{Ar}_{1}$ and aniline- $\mathrm{Ar}_{2}$ following excitation into bands $\operatorname{vdW}(1)$ and $\mathrm{vdW}(2)$, establishing that these two decay lifetimes are $10 \pm 2 \mathrm{~ns}$, while the lifetime of the $S_{1}$ origin of the bare aniline molecule is $12 \pm 2 \mathrm{~ns}$. Thus, no drastic reduction of the fluorescence quantum yield of the aniline- $\operatorname{Ar}_{n}(n=1$ and 2$)$ complexes is exhibited and it is improbable that effect (2) will prevail for the higher Ar complexes. We are just left with effect (3), which implies inhomogeneous broadening of the absorption spectra of the higher aniline- $\operatorname{Ar}_{n}(n \geqslant 3)$ van der Waals molecules.

We were unable to observe any distinct spectral features corresponding to aniline- $\mathrm{Kr}_{n}(n \geqslant 1)$ and aniline-Xe $(n \geqslant 1)$ complexes in the laser-induced fluoresence spectra of aniline seeded in supersonic expansions of $\mathrm{Kr}$ and of Xe up to $p=1.5 \mathrm{~atm}$. This negative result cannot be blamed on the kinetic effect (1), as figure 5 demonstrates efficient complexing of aniline in these diluents. In the case of van der Waals molecules containing $\mathrm{Kr}$ and $\mathrm{Xe}$, the external heavy atom effect enhances $S_{1} \rightarrow T_{1}$ intersystem crossing resulting in the reduction of the emission quantum yield [2,3]. Effect (2), together with the inhomogeneous broadening effect (3), may be responsible for our failure to observe any distinct spectral features of the complexes of aniline with $\mathrm{Kr}$ and with $\mathrm{Xe}$.

\section{Excited STATE ENERgetics of ANALINE- $\mathrm{Ne}_{1}$ AND ANILINE-Ar $\mathbf{r}_{1}$ COMPlexes}

The spectral shifts of the positions of the spectral features of the vibrationless $S_{0} \rightarrow S_{1}$ excitations of aniline $-R_{n}(R=\mathrm{Ne}$ and $\mathrm{Ar} ; n=1$ and 2$)$ complexes from the $0_{0}{ }^{\circ}$ band of the bare molecule provide direct information on the microscopic solvent shift of the electronic origin of the bare molecule. The spectral shift $\delta v$ for these complexes can roughly be expressed in terms of the additive contributions

$$
\delta \nu=\delta \nu_{\mathrm{R}}+\delta \nu_{\mathrm{DISP}}+\delta \nu_{\mathrm{DIDI}}
$$

where $\delta \nu_{\mathrm{R}}$ is due to short range repulsive interactions, which for intravalence excitations result in a (small) destabilization of $S_{1}$. $\delta \nu_{\text {DIS }}$ reflects a dispersive stabilization of the $S_{1}$ state. Finally, $\delta \nu_{\text {DIDI }}$ corresponds to the contribution from dipole-induced-dipole interactions. In van der Waals complexes of $\mathrm{He}$ the $\delta \nu_{R}$ contribution dominates, resulting in blue spectral shifts. For complexes consisting of alternant, unsubstituted, non-polar, aromatic hydrocarbons bound to heavy rare-gas atoms $\left(\mathrm{Ar}, \mathrm{Kr}\right.$ and Xe), the $\delta \nu_{\mathrm{DISP}}$ contribution to $\delta \nu$ is expected to dominate, being manifested by red spectral shifts which are roughly proportional to the polarizability $\alpha$ of the rare-gas atom $[9,31]$, that is

$$
\delta \nu_{\text {DISP }} \simeq-A \alpha
$$

where the parameter $A$ depends on the aniline $-R$ distance and on the geometry of the complex. In the case of the aniline $-R_{n}$ complexes, the electrostatic $\delta \nu_{\text {DIDI }}$ term is expected to contribute to the spectral shift. Without alluding to any numerical calculations, we can assert that for the DIDI contribution

$$
\delta \nu_{\mathrm{DIDI}} \simeq-B \alpha
$$


where the parameter $B$ depends on the aniline $-R$ distance and on the geometry of the complex. Furthermore, as the $S_{0} \rightarrow S_{1}$ transition of aniline results in the increase of the dipole moment, an extra stabilization of the $S_{1}$ state is exhibited.

The following features of the experimental spectral shifts are notable

(1) The spectral shifts for both aniline- $\mathrm{Ne}_{n}$ and for aniline- $\mathrm{Ar}_{n}(n=1$ and 2) are to lower energies. Thus, the contributions of the $\delta \nu_{\text {DISP }}$ and $\delta \nu_{\text {DIDI }}$ terms overwhelm the repulsive contribution $\delta \nu_{\mathbf{F}}$ to the spectral shifts.

(2) The ratio of the spectral shifts for aniline- $\mathrm{Ar}_{1}$ and of aniline- $-\mathrm{Ne}_{\mathbf{1}}$ is $\delta \nu(\mathrm{Ar}) / \delta \nu(\mathrm{Ne})=12$. Provided that the $\left(\delta \nu_{\mathrm{DISP}}+\delta \nu_{\mathrm{DIDI}}\right)$ terms provide a dominant contribution to $\delta \nu$, we expect on the basis of equations (4.1)-(4.3) that $\delta \nu(\mathrm{Ar}) / \delta \nu(\mathrm{Ne})=F \alpha(\mathrm{Ar}) / \alpha(\mathrm{Ne})$, where $F=[A(\mathrm{Ar})+$ $B(\mathrm{Ar})] /[A(\mathrm{Ne})+B(\mathrm{Ne})]$ and $\alpha(R)(R \equiv \mathrm{Ar}, \mathrm{Ne})$ is the polarizability of the $R$ atom. The distance dependence of the parameters $A$ and $B$ implies that $[8,9] F<1$, whereupon $\delta \nu(\mathrm{Ar}) / \delta \nu(\mathrm{Ne})<\alpha(\mathrm{Ar}) / \alpha(\mathrm{Ne})$. Using the experimental polarizability ratio $\alpha(\mathrm{Ar}) / \alpha(\mathrm{Ne})=4 \cdot 0$, we expect that $\delta \nu(\mathrm{Ar}) / \delta \nu(\mathrm{Ne})<4 \cdot 0$, in contrast to the experimental value of 12 for this ratio of the spectral shifts. The failure of this relation implies that the contribution of the repulsive term $\delta \nu_{\mathrm{R}}$ to the spectral shift of aniline- $\mathrm{Ne}_{1}$ complex is substantial.

(3) A large spectral shift is exhibited for the aniline- $\operatorname{Ar}_{n}(n=1$ and 2) complexes. As is apparent from table 4, the spectral shift for the $S_{0} \rightarrow S_{1}$ transition of aniline exerted by a single Ar atom considerably exceeds the corresponding spectral shifts of benzene and of alkyl benzenes.

Table 4. Experimental red spectral shifts $(\delta \nu)$ for the vibrationless excitation of $M-\mathrm{Ar}_{1}$ and of $M-\mathrm{Ar}_{2}$ van der Waals molecules.

\begin{tabular}{cccc}
\hline Molecule & $\delta \nu / \mathrm{cm}^{-1}$ & Reference & $f \dagger$ \\
\hline Aniline- $\mathrm{Ar}_{1}$ & $-58 \pm 3$ & Present work & $2 \cdot 2 \times 10^{-2}$ \\
Aniline- $\mathrm{Ar}_{2}$ & $-102 \pm 3$ & Present work & - \\
Toluene- $\mathrm{Ar}_{1}$ & $-26 \cdot 2 \pm 0 \cdot 8$ & {$[10]$} & $10^{-3}$ \\
Toluene- $_{2} r_{2}$ & $-48 \cdot 9 \pm 0 \cdot 8$ & {$[10]$} & - \\
Benzene-Ar & -20 & {$[11]$} & 0 \\
\hline
\end{tabular}

$\uparrow$ Electronic contribution to the oscillator strength for $S_{0} \rightarrow S_{1}$ transition of the bare aromatic molecule [34].

The red spectral shifts of the complexes of Ar with benzene and its derivatives (table 4$)$ are attributed to the dominating rôle of the $\left(\delta \nu_{\text {DISP }}+\delta \nu_{\text {DIDI }}\right)$ terms. A reliable numerical estimate of the dispersive contribution to $\delta \nu$ is an exceedingly difficult task. On the other hand, estimates of the first order electrostatic dipole-induced-dipole interactions are quite straightforward. Such a calculation of the $\delta \nu_{\text {DIDI }}$ for the complexes of aniline and of toluene with rare-gas atoms is presented in the Appendix. We have adopted a simple electrostatic model for the evaluation of $\delta \nu_{\text {DIDI }}$ using the valence electron charge density in the $S_{0}$ and $\mathrm{S}_{1}$ states. To specify the geometry of the aniline $-R_{1}$ complexes, we have utilized the results of model calculations [32] for benzene- $\mathrm{Ne}_{1}$, where the $\mathrm{Ne}$ atom is located at a distance $z_{R}=3 \cdot 12 \AA$ above the centre of the benzene ring and for 


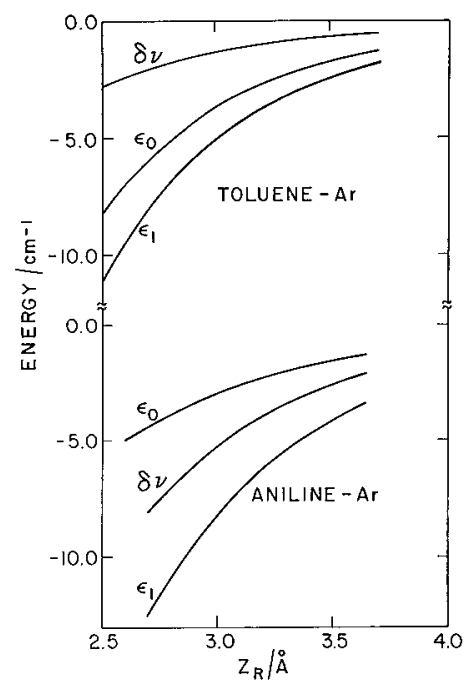

Figure 7. The electrostatic stabilization energies due to dipole-induced-dipole interaction, $\epsilon_{0}$ (equation (A 1)) and $\epsilon_{1}$ (equation (A 2)), together with the contribution to the spectral shift $\delta \nu_{\text {DIDI }}=\left(\epsilon_{1}-\epsilon_{0}\right)$ for aniline-Ar ${ }_{1}$ and toluene- $\operatorname{Ar}_{1}$ complexes.

benzene-Ar $r_{1}$, where $z_{\mathrm{R}}=3.48 \AA$. These calculations of the spectral shift (figure 7) of the $S_{0} \rightarrow S_{1}$ transition result in $\delta \nu_{\mathrm{DIDI}}=-2.6 \mathrm{~cm}^{-1}$ for aniline-Ar and $\delta \nu_{\text {DIDI }}=-1 \cdot 1 \mathrm{~cm}^{-1}$ for aniline- $\mathrm{Ne}_{1}$. These contributions from the dipoleinduced-dipole interactions are in the right direction. However, as $\delta \nu_{\text {DIDI }} / \delta \nu=$ 0.22 for aniline- $\mathrm{Ne}_{1}$ and $\delta \nu_{\mathrm{DIDI}} / \delta \nu \simeq 0.05$ for aniline- $\mathrm{A} \mathbf{r}_{1}$, the dipole-induceddipole interactions contribution provides a small contribution to the $\delta \nu$, while the major contribution to the red spectral shifts of these complexes originates from dispersive interactions.

We thus expect that the major contribution to the spectral shifts in the series benzene- $A r_{1}$, toluene- $A r_{1}$ and aniline- $\mathrm{Ar}_{1}$ (table 4) originates from dispersive interactions, reflecting the dependence of $\delta v$ on the features of the electronic excitation of the aromatic hydrocarbon. A very crude treatment of the dispersive interactions, based on the Pople/Longuet-Higgins approach [31], indicates that $\delta \nu_{\text {DISP }}$ will increase with increasing oscillator strength $f$ of the electronic transition. The dispersive stabilization is roughly given by (4.2) with $A=a+b f$, with $a$ and $b$ being again numerical constants which are determined by the geometry of the complex. The experimental data for the spectral shifts of the complexes of benzene and its derivatives (table 4 ) can be qualitatively accounted for in terms of the increase of the oscillator strength in the series benzene $<$ toluene $<$ aniline. The substantial spectral shift of aniline- $\mathrm{Ar}_{1}$ can be rationalized in terms of the large oscillator strength for the $S_{0} \rightarrow S_{1}$ transition of this molecule.

We are grateful to Dr. S. Leutwyler for his contribution to this work. Thanks are due to Professor R. E. Smalley for prepublication information and to Professor R. Bersohn for helpful discussions. This research was supported in part by the U.S.-Israel Binational Science Foundation, Jerusalem (No. 2641), by the Committee for Basic Research of the Israel National Academy of Sciences, Jerusalem (U.E.), and by the United States Army through its European Research Office. B. Dick would like to thank the Deutsche Forschungsgemeinschafs for a Research Fellowship. 


\section{APPENDIX}

\section{Calculation of THE DIPOLE-INDUCED-Dipole INTERACTIONS}

An electrostatic model was advanced to provide a semiquantitative description of the contribution of dipole-induced-dipole interactions to the spectral shift of the van der Waals complexes of Ar with polar substituted benzenes. We have calculated $\delta \nu_{\text {DIDI }}$ for the $S_{0} \rightarrow S_{1}$ transition of toluene- $R_{1}$ and for aniline- $R_{1}$ ( $R=\mathrm{Ne}$ and $\mathrm{Ar}$ ) complexes, considering the interaction of the rare-gas atom with the valence electron charge distribution on the atoms of the aromatic molecule. The interaction energy was calculated separately for the $S_{1}$ excited state and for the $S_{0}$ ground state, which differ in their valence electron charge distribution. The electrostatic stabilization energies in the two electronic states of aniline-Ar are

$$
\epsilon_{0}=-\frac{\alpha e^{2}}{2} \mathbf{F}^{(0)} \cdot \mathbf{F}^{(0)}
$$

for the $S_{0}$ state and

$$
\epsilon_{1}=-\frac{\alpha e^{2}}{2} \mathbf{F}^{(1)} \cdot \mathbf{F}^{(1)}
$$

for the $S_{1}$ state. Here $\alpha$ is the polarizability of the rare-gas atom, while $\mathbf{F}^{(0)}$ and $F^{(1)}$ represent the electrostatic force exerted by the valence charge distribution of the aniline molecule on the rare-gas atom in the electronic states $S_{0}$ and $S_{1}$, respectively. These can readily be expressed in terms of the charge distribution $e_{j}{ }^{(0)}$ and $e_{j}{ }^{(1)}$ on the $j$ th atom of the aromatic molecule in the $S_{0}$ and $S_{1}$ states, respectively, and by the static geometry of the complex, which is specified by coordinates $\left(x_{\mathrm{R}}, y_{\mathrm{R}}\right.$ and $\left.z_{\mathrm{R}}\right)$ of the rare-gas atom, and by the atomic coordinates $\left(x_{j}, y_{j}\right.$ and $\left.z_{j}\right)$ of the substituted benzene molecule. The explicit expressions are

$$
\epsilon_{\eta}=-\frac{\alpha e^{2}}{2}\left(F_{x}(\eta)^{2}+F_{y}(\eta)^{2}+F_{z}^{(\eta)^{2}}\right) ; \quad \eta=0,1
$$

where

$$
\begin{aligned}
F_{\xi}(\eta) & =\sum_{j=1} \frac{\left(e_{n}{ }^{(\eta)} / e\right)}{r_{j}{ }^{3}}\left(\xi_{j}-\xi_{\mathrm{R}}\right) ; \quad \xi=x, y, z, \quad \eta=0,1 \\
r_{j} & =\left[\left(x_{j}-x_{\mathrm{R}}\right)^{2}+\left(y_{j}-y_{\mathrm{R}}\right)^{2}+\left(z_{j}-z_{\mathrm{R}}\right)^{2}\right]^{1 / 2} .
\end{aligned}
$$

The $\pi$ electron charge distribution for the $S_{0}\left({ }^{1} A_{1}\right)$ and $S_{1}\left({ }^{1} B_{2}\right)$ states of toluene and of aniline were obtained by a CI calculation [33], which contained the lowest-lying 200 singly-excited and doubly-excited configurations. The calculated ${ }^{1} A_{1} \rightarrow{ }^{1} B_{2}$ excitation energies are $4.72 \mathrm{eV}$ for aniline and $4.55 \mathrm{eV}$ for toluene, while the dipole moments for aniline were calculated as $\mu\left(S_{0}\right)=$ 0.83 Debye and $\mu\left(S_{1}\right)=1.90$ Debye. These calculated values are lower than the experimental data [13], $\mu\left(S_{0}\right)=1.53$ Debye and $\mu\left(S_{1}\right)=2.38 \pm 0.1$ Debye. Nevertheless, we prefer to use the calculated valence electron charge densities for the evaluation of $\delta \nu_{\text {DIDI }}$, rather than to approximate the large molecule by a point dipole, whose magnitude in $S_{0}$ and in $S_{1}$ is given by the experimental values. A similar CI calculation for toluene yielded $\mu\left(S_{0}\right)=0.43$ Debye and $\mu\left(S_{1}\right)=0.69$ Debye. In figure 7 we present the calculated energies $\epsilon_{0}$ and $\epsilon_{1}$, together with the spectral shift $\delta \nu_{\mathrm{DIDI}}=\left(\epsilon_{1}-\epsilon_{0}\right)$ for toluene-Ar$r_{1}$ and aniline-Ar complexes with the Ar atom located at the distance $z_{\mathrm{R}}$ above the centre of the 
aromatic ring. Taking $z_{\mathrm{R}}=3.48 \AA$ for the Ar complexes [32], electrostatic contributions are $\delta \nu_{\text {DIDI }}=-0.7 \mathrm{~cm}^{-1}$ for toluene- $A r_{1}$ and $\delta \nu_{\text {DIDI }}=-2.6 \mathrm{~cm}^{-1}$ for aniline-Ar $\mathbf{r}_{1}$. Data for complexes containing other rare gases can be obtained by scaling of the data of figure 7 by the appropriate ratio of the polarizabilities. From these results we conclude that the contribution of the dipole-induceddipole interactions, both to the ground state stabilization energy and to the spectral shift, are small relative to the contribution originating from dispersive interactions.

\section{REFERENCES}

[1] Amirav, A., Even, U., and Jortner, J., 1979, Chem. Phys. Lett., 67, 9.

[2] Amirav, A., Even, U., and Jortner, J., 1981, f. chem. Phys., 75, 2489.

[3] Amirav, A., Even, U., and Jortner, J., 1981, F. phys. Chem., 85, 309.

[4] Amirav, A., Even, U., and Jortner, J., 1981, F. chem. Phys., 74, 3745.

[5] Amirav, A., Even, U., and Jortner, J., 1982, Chem. Phys., 67, 1.

[6] Leutwyler, S., Even, U., and Jortner, J., 1982, Chem. Phys. Lett., 86, 439.

[7] Heyes, 'T. R., Henke, W., Selzle, H. L., and Schlag, E. W., 1980, Chem. Phys. Lett., $77,19$.

[8] Ondrechen, M. J., Berkovitch-Yellin, Z., and Jortner, J., 1981, F. Am. chem. Soc., $103,6586$.

[9] Even, U., Amirav, A., Leutwyler, S., Ondrechen, M. J., Berkowitch-Yellin, Z., and JorTner, J., 1982, Discuss. Faraday Soc., 73, 153.

[10] Hopkins, J. B., and Smalley, R. E., Private communication and to be published.

[11] Fung, K. H., Selzle, H. L., and Schlag, E. W., 1981, Z. Naturf. (a), 36, 1338.

[12] Fung, K. H., Henke, W. E., Selzle, H. L., and SchlaG, E. W., 1981, Ұ. phys. Chem., $85,3560$.

[13] Lombardi, J. R., 1969, F. chem. Phys., 50, 3780.

[14] Even, U., Magen, J., and Jortner, J., 1982, Chem. Phys. Lett., 88, 131.

[15] Chernoff, D. A., and Rice, S. A., 1979, Ұ. chem. Phys., 70, 2511.

[16] Quack, M., and StockBenger, M., 1972, 7. molec. Spectrosc., 43, 87.

[17] Mirami, N., Hiraya, A., Fujiwara, I., and Ito, M., 1980, Chem. Phys. Lett., 74, 531.

[18] Dietz, T. G., Duncan, M. A., Liverman, M. G., and Smalley, R. E., 1980, Chem. Phys. Lett., 70, 246.

[19] Powers, D. E., Hopkins, J. B., and Smalley, R. E., 1980, F. chem. Phys., 72, 5721.

[20] Ashrenas, A., and Sherman, F. S., 1966, Rarefied Gas Dynamics, Vol. 2, edited by J. H. de Leeuw (Academic Press), p. 84.

[21] McClelland, G. M., Saenger, K. L., Valentino, J. J., and Herschbach, D. R., 1979, F. phys. Chem., 83, 947.

[22] Amirav, A., Even, U., Jortner, J., Birss, F. W., and Ramsay, D. A., 1983, Can. F. Phys., 61, 278.

[23] Birss, F. W., Brown, J. M., Cole, A. R. H., Lofthus, A., Krishnamachari, S. L. N. G., Osborne, G. A., Paldus, J., Ramsay, D. A., and Watman, L., 1970, Can. F. Phys., 48, 1230.

[24] Birss, F. W., Colson, S. D., and Ramsay, D. A., 1973, Can. F. Phys., 51, 1031.

[25] Christoffersen, J., Hollas, J. M., and Kirby, G. H., 1969, Molec. Phys., 16, 441.

[26] Amirav, A., Even, U., and Jortner, J., 1980, Chem. Phys., 51, 31.

[27] Larsen, N. W., Hansen, E. L., and Nicolaisen, F. M., 1976, Chem. Phys. Lett., 43, 584.

[28] Leutwyler, S., and Even, U., 1981, Chem. Phys. Lett., 81, 578.

[29] Smalley, R. E., Wharton, L., and Levy, D. H., 1977, Accts chem. Res., 10, 139.

[30] Bunker, D. L., 1962, 7. chem. Phys., 32, 1001.

[31] Longuet-Higgins, H. C., and Pople, J. A., 1957, F. chem. Phys., 27, 192.

[32] Jortner, J., and Berkowitch-YelLin, Z. (unpublished).

[33] Dick, B., 1981, Ph.D. Thesis, University of Köln.

[34] Petruska, J., 1961, F. chem. Phys., 34, 1120. 